\title{
Inhibition of aortic wall calcification in bioprosthetic heart valves by ethanol pretreatment: Biochemical and biophysical mechanisms
}

\author{
Chi-Hyun Lee, ${ }^{1}$ Narendra Vyavahare, ${ }^{2}$ Robert Zand, ${ }^{3}$ Howard Kruth,${ }^{4}$ Frederick J. Schoen, ${ }^{5}$ Richard Bianco, ${ }^{6}$ \\ Robert J. Levy ${ }^{2}$ \\ ${ }^{1}$ Department of Pediatrics and Communicable Diseases, The University of Michigan, Ann Arbor, Michigan 48109 \\ ${ }^{2}$ The Joseph Stokes, Jr., Research Institute, Children's Hospital of Philadelphia, Abramson Pediatric Research Center, \\ 1107B, 34th Street and Civic Center Blvd., Philadelphia, Pennsylvania 19104 \\ ${ }^{3}$ Department of Biochemistry and Biophysics, University of Michigan, Ann Arbor, Michigan 48109 \\ ${ }^{4}$ NHLBI, Bethesda, Maryland \\ ${ }^{5}$ Brigham and Women's Hospital and Harvard Medical School, Boston, Massachusetts \\ ${ }^{6}$ Department of Surgery, University of Minnesota Medical School, Minnesota
}

Received 16 September 1997; 20 February 1998

\begin{abstract}
The effectiveness of ethanol pretreatment on preventing calcification of glutaraldehyde-fixed porcine aortic bioprosthetic heart valve (BPHV) cusps was previously demonstrated, and the mechanism of action of ethanol was attributed in part to both lipid removal and a specific collagen conformational change. In the present work, the effect of ethanol pretreatment on BPHV aortic wall calcification was investigated using both rat subdermal and sheep circulatory implants. Ethanol pretreatment significantly inhibited calcification of BPHV aortic wall, but with less than complete inhibition. The maximum inhibition of calcification of BPHV aortic wall was achieved using an $80 \%$ ethanol pretreatment; calcium levels were $71.80 \pm 8.45 \mu \mathrm{g} / \mathrm{mg}$ with $80 \%$ ethanol pretreatment compared to the control calcium level of 129.90 $\pm 7.24 \mu \mathrm{g} / \mathrm{mg}(p=0.001)$. Increasing the duration of ethanol exposure did not significantly improve the inhibitory effect of ethanol on aortic wall calcification. In the sheep circulatory implants, ethanol pretreatment partly prevented BPHV aortic wall calcification with a calcium level of $28.02 \pm 4.42$ $\mu \mathrm{g} / \mathrm{mg}$ compared to the control calcium level of $56.35 \pm 6.14$
\end{abstract}

$\mu \mathrm{g} / \mathrm{mg}(p=0.004)$. Infrared spectroscopy (ATR-FTIR) studies of ethanol-pretreated BPHV aortic wall (vs. control) demonstrated a significant change in protein structure due to ethanol pretreatment. The water content of the aortic wall tissue and the spin-lattice relaxation times $\left(T_{1}\right)$ as assessed by proton nuclear magnetic resonance spectroscopy did not change significantly owing to ethanol pretreatment. The optimum condition of $80 \%$ ethanol pretreatment almost completely extracted both phospholipids and cholesterol from the aortic wall; despite this, significant calcification occurred. In conclusion, these results clearly demonstrate that ethanol pretreatment is significantly but only partially effective for inhibition of calcification of BPHV aortic wall and this effect may be due in part to lipid extraction and protein structure changes caused by ethanol. It is hypothesized that ethanol pretreatment may be of benefit for preventing bioprosthetic aortic wall calcification only in synergistic combination with another agent. (C) 1998 John Wiley \& Sons, Inc. J Biomed Mater Res, 42, 30-37, 1998.

\section{INTRODUCTION}

Calcification is the most frequent cause of the clinical failure of bioprosthetic heart valve (BPHV) replacements fabricated from porcine aortic valves

Correspondence to: R. J. Levy

Contract grant sponsor: National Institutes of Health; Contract grant number: HL 38118

Contract grant sponsor: The Children's Hospital of Philadelphia (to R. J. Levy)

(c) 1998 John Wiley \& Sons, Inc.

CCC 0021-9304/98/010030-08 crosslinked with glutaraldehyde. ${ }^{1}$ Calcification refers to the deposition of calcium phosphates and associated minerals in the BPHV tissues. This calcific degeneration is usually followed by stenosis or cuspal tearing with regurgitation, and can result in prosthesis failure. Although calcification is always pathological in the cardiovascular system, calcium phosphate mineral deposition and resorption occur in the course of normal bone and tooth development and metabolism. Since cell-oriented calcification and collagen calcification are prominent pathophysiologic features of bioprosthetic calcification, inhibition of both disease processes is necessary for therapeutic efficacy. 
Recently, the calcification of the bioprosthetic aortic wall has been studied owing to the increasing use of the stentless BPHV, ${ }^{2}$ which has a relatively large segment of exposed aortic wall, compared to the stented valve, in which virtually no aortic wall is exposed outside the stent. Thus, bioprosthetic aortic wall calcification has the potential to compromise the clinical function of the stentless BPHV. The effectiveness of ethanol pretreatment on inhibition of BPHV cusp calcification was previously demonstrated. ${ }^{3}$ However, the effects of ethanol pretreatment on calcification of BPHV aortic wall have not been investigated until the present study.

Our objective was to study the inhibitory effect of ethanol pretreatment on the calcification of bioprosthetic aortic wall. Using rat subdermal implants and sheep mitral valve replacement implants for assessing ethanol inhibition of the calcification of BPHV aortic wall, we studied the effects of various exposure conditions (i.e., various ethanol concentrations and durations of pretreatment) for ethanol pretreatment on calcification of bioprosthetic aortic wall. We also sought to determine the mechanisms responsible for the inhibition of calcification by ethanol pretreatment by comparing bioprosthetic aortic wall to cusp.

\section{MATERIALS AND METHODS}

\section{Materials}

Fresh porcine aortic valves, obtained at a slaughterhouse and transported to the laboratory on ice within $24 \mathrm{~h}$, were provided by St. Jude Medical Co. (Minneapolis, MN). The aortic root was dissected and rinsed with copious amounts of sterile saline to remove adherent blood, and then dissected free of extraneous tissue. Glutaraldehyde fixation procedures and ethanol exposure conditions (see below) were previously described. ${ }^{3}$ Aortic walls were crosslinked with $0.6 \%$ glutaraldehyde (Polysciences, Warrington, PA)/ $0.05 M$ HEPES $\mathrm{pH} 7.4 / 0.14 \mathrm{M} \mathrm{NaCl}$ at room temperature for $24 \mathrm{~h}$, and subsequently stored in the same buffer with $0.2 \%$ glutaraldehyde for at least 7 days to achieve complete fixation prior to use.

\section{Ethanol pretreatment of the aortic wall}

For the evaluation of concentration-dependent effects of ethanol on calcification of bioprosthetic heart valves, glutaraldehyde-preserved porcine aortic wall samples were stored in each of the graded ethanol solutions $[0,50,70,80$, and 90 $\mathrm{v} / \mathrm{v}$ in $0.05 M$ HEPES buffer ( $\mathrm{pH} 7.4$ ) and $100 \%$ unbuffered] (10 $\mathrm{mL}$ for each aortic wall sample, with a typical size of $1 \times$ $1 \mathrm{~cm}^{2}$ ) for $24 \mathrm{~h}$ at room temperature. Samples were rinsed free of ethanol and stored in an excess of sterile saline for 24 $\mathrm{h}$ prior to implantation. For the control tissues, glutaraldehyde-preserved porcine aortic wall specimens were treated with 0.05M HEPES buffer ( $\mathrm{pH}$ 7.4) for $24 \mathrm{~h}$ at room tem- perature and then rinsed with sterile saline for $24 \mathrm{~h}$. An ethanol solution $(80 \%)$ was used for the time exposure experiments $(1,3$, and 7 days) under the same buffer conditions as used in the concentration dependence study.

\section{Rat subdermal implantation}

Male Sprague-Dawley rats (about 1 month old) were anesthetized by intramuscular injections of ketamine $\mathrm{HCl}$ (Ketaset ${ }^{\circledR}$; Fort Dodge Lab, Fort Dodge, IA) and xylazine (Rompun ${ }^{\circledR}$; Miles, Shawnee Mission, KS). Samples were then implanted into subcutaneous pouches dissected into the dorsal scapular area. Each rat received two to four implants. Aortic wall samples were explanted 8 weeks after implantation. Animals were euthanized by $\mathrm{CO}_{2}$ asphyxiation before explantation.

\section{Sheep mitral valve replacements}

Clinical grade porcine aortic valve bioprostheses (St. Jude Medical; size $25 \mathrm{~mm}$ ) were implanted in the mitral position of Dorset or Columbian crossbred female sheep (28-38 kg, 3-4 months old) as previously described. ${ }^{3}$ Samples were implanted into the mitral position under halothane/ isofluorane inhalation anesthesia via a standard left thoracotomy through the fourth intercostal space with the aid of cardiopulmonary bypass, as previously described. ${ }^{4}$ Subcutaneous heparin sulfate (100 U) was given twice a day for 2 days; Proxicam (10 mg orally) was given once each day beginning on postoperative day 1 . Sheep (20 animals) used for this study were fed diets containing the recommended daily calcium intake for young developing sheep. Approximately 2 weeks after the operation, the sheep were transferred to a farm. All implants lasted for 150 days and were terminated by elective euthanasia with an overdose of pentobarbital.

\section{Calcium analyses}

Aortic wall samples explanted from either rats or sheep were washed with an excess of saline followed by distilled water. Samples were lyophilized and weighed. Each sample $(15-40 \mathrm{mg})$ was hydrolyzed in $1 \mathrm{~mL}$ of $6 \mathrm{~N} \mathrm{HCl}$ in a test tube at $70^{\circ} \mathrm{C}$ for $6-8 \mathrm{~h}$ or until dissolution, as previously published. ${ }^{3}$ The solution was then completely evaporated at $70^{\circ} \mathrm{C}$ with a continuous flow of nitrogen in the tube. The residue in the test tube was dissolved in $1 \mathrm{~mL}$ of $0.01 \mathrm{~N} \mathrm{HCl}$. The calcium content of these samples was measured using atomic absorption (AA) spectroscopy as previously described. $^{3}$

\section{Phosphorus analyses}

Inorganic phosphorus levels were determined on aliquots of the same preparations as used for calcium analyses. The phosphorous content of samples was determined using a molybdate complexation method, ${ }^{5}$ and the phosphorous 
concentration as reflected by the molybdate complex was measured at $820 \mathrm{~nm}$ using a Perkin Elmer Lambda 3B UV/ VIS Spectrophotometer (Perkin-Elmer Co., Norwalk, CT). Standard curves were generated using a commercially available phosphorous standard (Sigma, St Louis, MO).

\section{Infrared spectroscopy of aortic wall}

Unimplanted BPHV aortic wall samples were used for infrared spectroscopy studies. A Perkin Elmer FTIR spectrophotometer (Model 1740) equipped with a horizontal attenuated total reflectance (ATR) ZnSe cell (45, ATR, Spectra Tech) was used. The hydrated tissues were placed on the ATR cell and compressed with a sponge to obtain good surface contact. The hydrated tissues were scanned from 4000 to $450 \mathrm{~cm}^{-1}$ for 50 scans with a resolution of $4 \mathrm{~cm}^{-1}$. Water was used as a reference and was subtracted from the tissue spectra. The spectra were deconvoluted in the region of $1800-1000 \mathrm{~cm}^{-1}$ with the Perkin Elmer software program to resolve the spectrum.

\section{Determination of equilibrium water content of the aortic wall tissue}

Glutaraldehyde-crosslinked aortic wall (control and ethanol pretreated) was weighed in the wet state after blotting on filter paper to remove surface water, and then lyophilized. The dry weight of the cusp samples was obtained after lyophilization and the percent water content of the specimens was calculated by the following formula:

$\%$ Water content $=100 \times($ Wet weight - Dry weight $)$ / Wet weight.

\section{Solid-state proton nuclear magnetic resonance (NMR) study}

Four to five aortic wall samples $(0.5 \times 0.5 \mathrm{~cm})$ from each group (control and ethanol pretreated) that were equilibrated in distilled water were taken out and blotted on filter paper to remove surface water. These tissue samples were then placed in an NMR tube (10 $\mathrm{mm}$ in diameter). The samples were analyzed with NMR at $40^{\circ} \mathrm{C}$. A solid-state NMR spectrometer [Bruker Minispec, PC20; Bruker Spectrospin (Canada) Ltd., Milton, Ontario, Canada] was used to obtain spin-lattice relaxation times $\left(T_{1}\right) \cdot{ }^{6,7} T_{1}$ values were obtained from an inversion recovery pulse sequence (180TD-90), in which the amplitude of the free induction decay (FID) was measured as a function of the interpulse distance TD. TD was varied from $1 \mathrm{~ms}$ to at least five times $T_{1}$. The spectrometer was calibrated for each sample to assure an accurate duration of $90^{\circ}$ and $180^{\circ}$ radiofrequency pulses.

\section{Lipid analysis}

Glutaraldehyde-fixed aortic wall samples were divided into two groups. One group of samples was pretreated with
HEPES buffer for $24 \mathrm{~h}$ (control), while the other group of samples was pretreated with $80 \%$ ethanol in HEPES buffer for $24 \mathrm{~h}$. After the pretreatment, samples were washed with water and lyophilized. The weighed samples were then extracted with chloroform/methanol 2:1 (vol/vol). ${ }^{8}$ Total cholesterol was determined enzymatically with fluorometric detection. ${ }^{9}$ Total phospholipid phosphorus was determined by the modified Fiske-Subbarow method ${ }^{10}$ with a FiskeSubbarow reagent obtained from Fisher Scientific Co. Total cholesterol and phospholipids were expressed as nanomoles per milligram of dry tissue.

\section{Statistical analysis}

In most cases (unless specified otherwise), at least 10 different samples were used for each group. Data were expressed as the mean \pm standard error of mean (SEM). Statistical significance was obtained by Student $t$ test (unpaired), and $p$ values of 0.05 were termed significant.

\section{RESULTS}

\section{Rat subdermal implants}

A concentration-dependent effect of ethanol pretreatment in reducing the calcium and phosphorus content of BPHV aortic wall specimens implanted for 8 weeks in the rat subcutaneous model is shown in Figure 1. Ethanol pretreatment significantly $(p=0.001)$

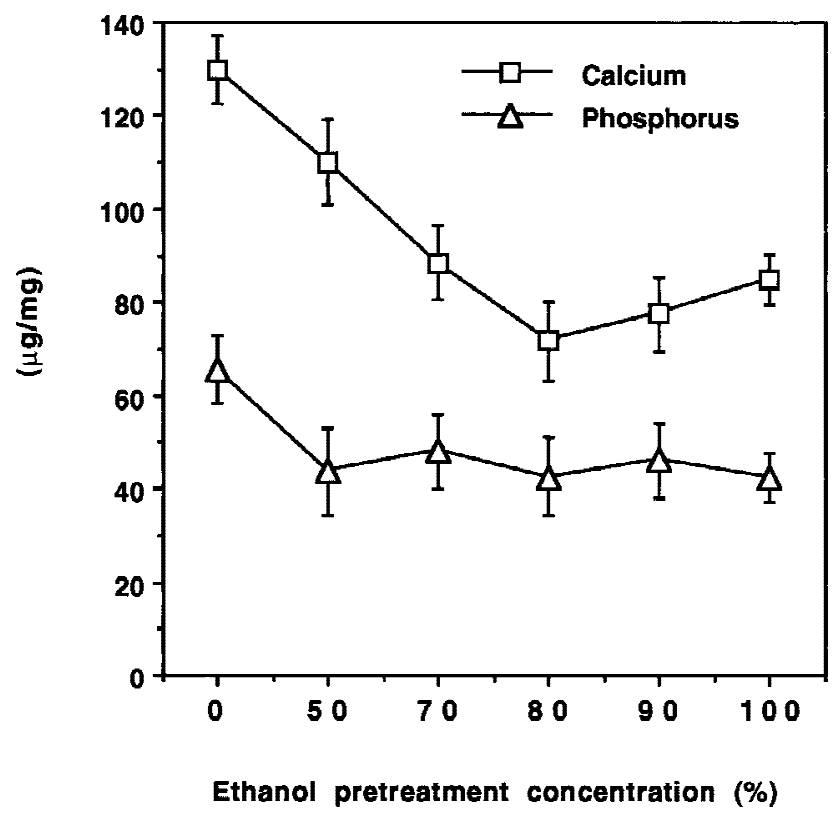

Figure 1. The effect of the concentration of the ethanol pretreatment solution on calcium and phosphorus content of glutaraldehyde crosslinked porcine aortic wall; results of 8week rat subdermal implants. 
inhibited BPHV aortic wall calcification compared to the controls, but with less than complete inhibition. The maximum inhibition of calcification of glutaraldehyde-preserved porcine bioprosthetic aortic wall was achieved using $80 \%$ ethanol solution with a resultant calcium level of $71.80 \pm 8.45 \mu \mathrm{g} / \mathrm{mg}$ for the tissue compared to a calcium level of $129.90 \pm 7.24 \mu \mathrm{g} / \mathrm{mg}$ of the control tissues (Fig. 1). Ethanol pretreatment caused a significant decrease in aortic wall phosphorous content versus control, with a phosphorous level of $42.55 \pm 5.01 \mu \mathrm{g} / \mathrm{mg}$ of the tissue treated with $80 \%$ ethanol compared to the control tissues with a phosphorous level of $65.71 \pm 7.65 \mu \mathrm{g} / \mathrm{mg}$ (Fig. 1). The inhibitory effects of calcification of aortic wall pretreated with $90 \%$ and $100 \%$ ethanol were slightly less than that with $80 \%$ ethanol, but were not significantly different. The relationship between molar ratio of [Ca]/ $[\mathrm{P}]$ in aortic wall implanted for 8 weeks and ethanol concentrations is shown in Table I. The ratios were relatively constant through the tested concentration range of ethanol and were comparable to calcified control implants. The $[\mathrm{Ca}] /[\mathrm{P}]$ ratio is consistent with hydroxyapatite as the predominant mineral phase.

There was no significant difference between longterm (up to 1 week) exposure to ethanol solution (80\%) versus $24 \mathrm{~h}$ in terms of inhibition of calcification of BPHV aortic wall (Fig. 2). Although the $80 \%$ ethanol pretreatment for 7 days did reduce the calcium levels as compared to 1-day pretreatment (from $71.8 \pm 5.0$ to $63.0 \pm 6.15 \mu \mathrm{g} / \mathrm{mg}$ ), the difference was not significant.

\section{Sheep mitral valve replacements}

As shown in Table II, ethanol pretreatment significantly inhibited aortic wall calcification compared to controls $(\mathrm{Ca}=28.02 \pm 4.42$ vs. $56.65 \pm 6.14 \mu \mathrm{g} / \mathrm{mg})$, but with less than complete inhibition. Calcium levels in ethanol-pretreated aortic wall were also significantly different from unimplanted aortic wall $(\mathrm{Ca}=28.02 \pm$ 4.42 vs. $0.72 \pm 0.08 \mu \mathrm{g} / \mathrm{mg}$ ).

TABLE I

$\mathrm{Ca} / \mathrm{P}$ Molar Ratios Obtained for Ethanol-Pretreated Bioprosthetic Aortic Wall after 8 Weeks of Rat Subdermal Implantation (Pretreatment Time $24 \mathrm{~h}$ )

\begin{tabular}{cc}
\hline Pretreatment Condition & $\mathrm{Ca} / \mathrm{P}$ Molar Ratio \\
\hline Control & 1.58 \\
$50 \%$ ethanol & 2.01 \\
$70 \%$ ethanol & 1.47 \\
$80 \%$ ethanol & 1.35 \\
$90 \%$ ethanol & 1.35 \\
$100 \%$ ethanol & 1.60 \\
\hline
\end{tabular}

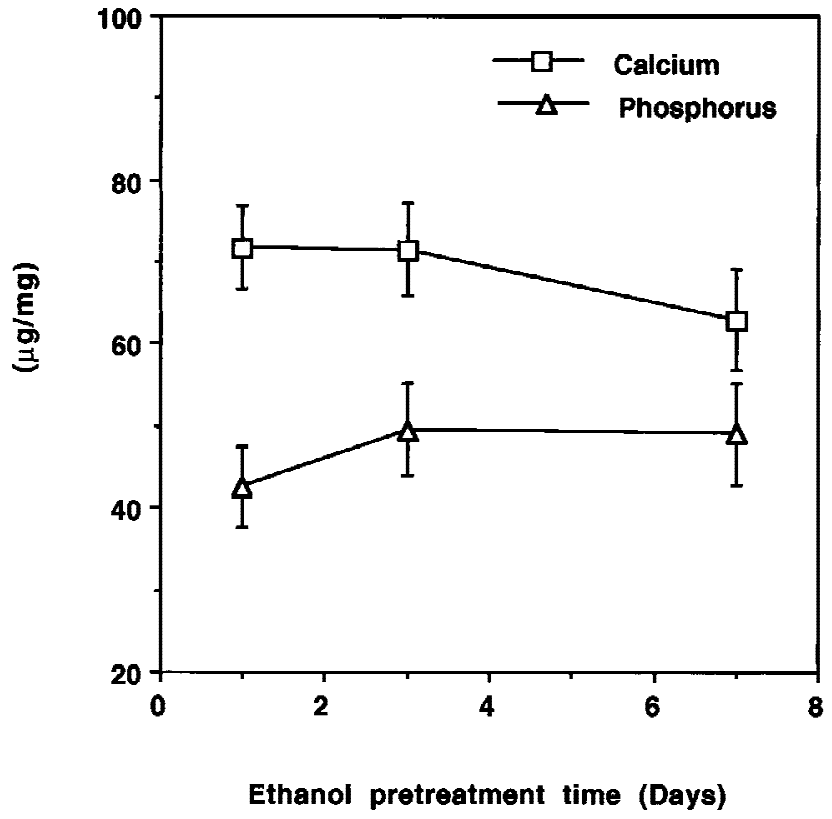

Figure 2. The effect of extended $80 \%$ ethanol pretreatments (1, 3, and 7 days) on calcium and phosphorus content of glutaraldehyde crosslinked porcine aortic wall; results of 8week rat subdermal implants.

Protein conformational changes as assessed by attenuated total reflectance-Fourier transform infrared spectroscopy (ATR-FTIR)

The overlay of deconvoluted ATR-FTIR spectra of glutaraldehyde-crosslinked aortic wall (control) and $80 \%$ ethanol pretreated aortic wall is shown in Figure 3. For control BPHV aortic wall samples not exposed to ethanol, the amide carbonyl stretching region (amide I, 1700-1600 $\mathrm{cm}^{-1}$ ) was resolved into multiple bands, including a $1651-\mathrm{cm}^{-1}$ band with a shoulder at 1659, a 1636 band, and a 1619 band. After the ethanol pretreatment, major changes were seen in the amide I region. A prominent band at $1619 \mathrm{~cm}^{-1}$ seen in controls was almost completely absent. There was a downward shift in the other amide bands, with the $1651-\mathrm{cm}^{-1}$ band shifted to $1645 \mathrm{~cm}^{-1}$ and the 1636 $\mathrm{cm}^{-1}$ band shifted to $1633 \mathrm{~cm}^{-1}$. Other major changes in the spectra were seen in the $1100-1000-\mathrm{cm}^{-1}$ region (CO stretching region of the carbohydrate residues). ${ }^{11}$

TABLE II

Effect of Ethanol Pretreatment on Inhibition of Bioprosthetic Calcification after Implantation in Sheep for 150 Days

\begin{tabular}{lc}
\hline \multicolumn{1}{c}{ Group } & Calcium $(\mu \mathrm{g} / \mathrm{mg}$, mean $\pm \mathrm{SEM})$ \\
\hline Control & $56.65 \pm 6.14^{*}$ \\
Ethanol $(80 \%)$ treated & $28.02 \pm 4.42^{*}$ \\
Unimplanted & $0.72 \pm 0.08^{*}$ \\
\hline
\end{tabular}

${ }^{*} \mathrm{p}=0.001$. 


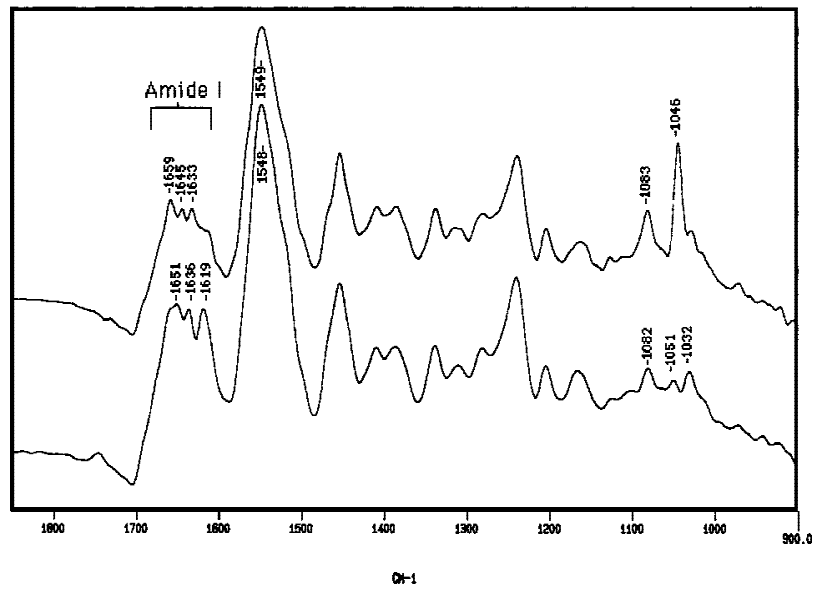

Figure 3. Overlay of deconvoluted Fourier transform infrared spectra for control (lower) and 80\% ethanolpretreated (upper) porcine bioprosthetic aortic wall. Note the changes in the amide I region $\left(1680-1600 \mathrm{~cm}^{-1}\right)$ and in the lower region $\left(1100-1000 \mathrm{~cm}^{-1}\right)$ of the spectra after ethanol pretreatment for $24 \mathrm{~h}$.

A new sharp band appeared at $1046 \mathrm{~cm}^{-1}$ after ethanol pretreatment.

\section{Proton NMR studies and lipid extraction results}

The $T_{1}$ values for aortic wall tissue were in the range of 370-380 ms (Fig. 4). The $T_{1}$ values did not differ significantly after the various ethanol pretreatments. Aortic wall water content ranged from $70 \%$ to $77 \%$ and did not show any appreciable change after the various ethanol pretreatments (Fig. 4). In contrast, a marked decrease in the proton NMR relaxation time $\left(T_{1}\right)$ associated with increasing concentrations of the
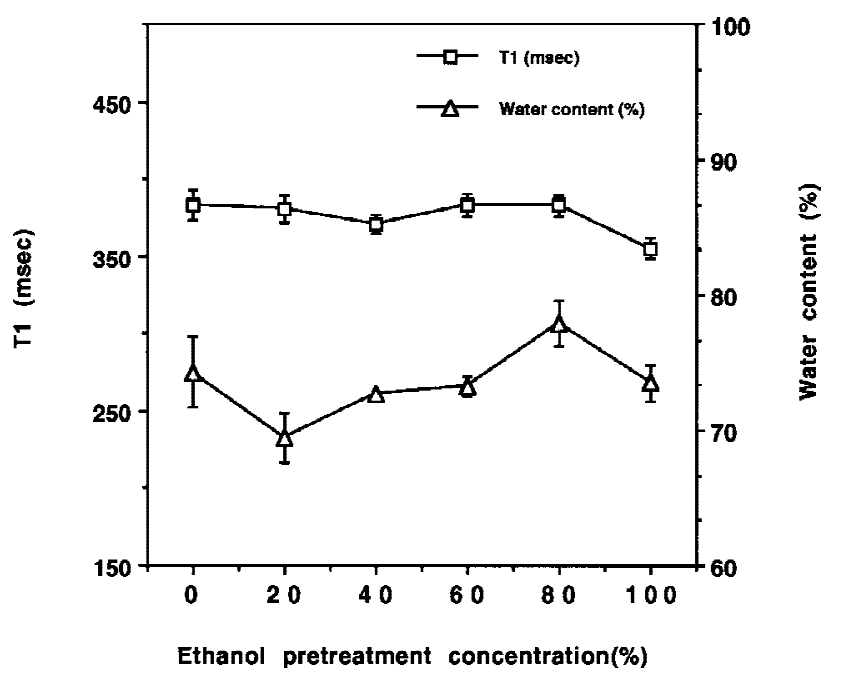

Figure 4. The effect of $80 \%$ ethanol pretreatment for $24 \mathrm{~h}$ on proton NMR spin-lattice relaxation times $\left(T_{1}\right)$ and water content of glutaraldehyde-crosslinked porcine aortic wall. ethanol pretreatment solution was observed previously with bioprosthetic cusps ${ }^{3}$ (Fig. 5), indicating that markedly different water interactions took place after the ethanol pretreatments of cusps versus BPHV aortic wall. As shown in Table III, the $80 \%$ ethanol pretreatment extracted almost all phospholipids and cholesterol from the aortic wall samples.

\section{DISCUSSION}

Previous work by our group demonstrated that pretreatment of bioprosthetic aortic cusps with ethanol inhibited calcification in both subdermal implants and sheep mitral valve replacements. ${ }^{3}$ The mechanism of inhibition was attributed to an interaction of lipid extraction with a specific collagen structural change evident by a permanent alteration in the amide I carbonyl stretching region of the infrared spectra. ${ }^{3}$

Although ethanol pretreatment significantly inhibited BPHV aortic wall calcification in the present studies, the effect was incomplete, unlike the complete inhibition of cusp calcification seen with ethanol pretreatment. Nevertheless, the effect was much better than that observed with other anticalcification agents currently of interest for clinical use. Specifically, amino oleic acid pretreatment, while significantly inhibiting bioprosthetic cusp mineralization, had little effect on inhibiting calcification of aortic wall in circulatory implants. ${ }^{2}$ However, previous rat implant studies by our group have demonstrated that aortic wall calcification can be significantly inhibited through the use of a number of agents of interest. For example, pretreatment with aluminum chloride or bisphosphonates significantly inhibited bioprosthetic aortic wall

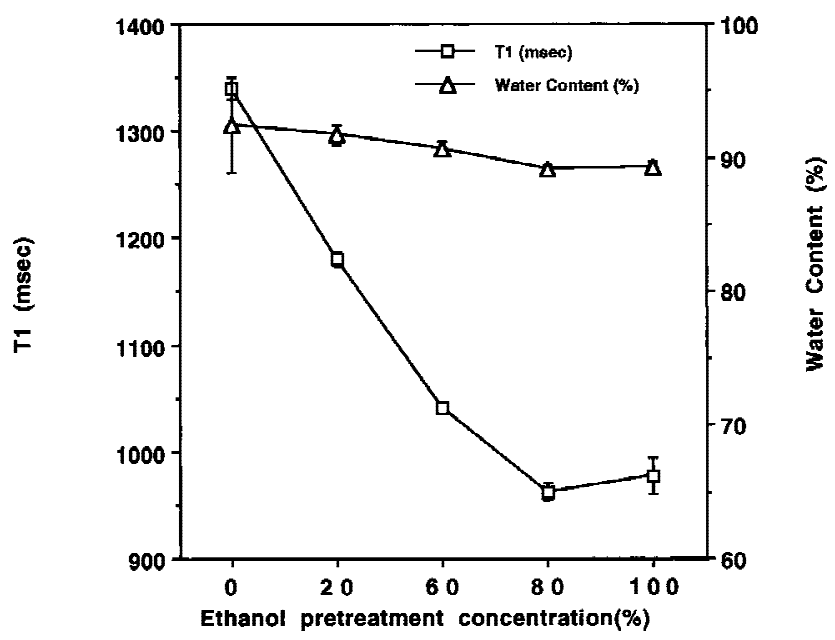

Figure 5. The effect of $80 \%$ ethanol pretreatment for $24 \mathrm{~h}$ on proton NMR spin-lattice relaxation times $\left(T_{1}\right)$ and water content of glutaraldehyde-crosslinked porcine cusps (data from Vyavahare et $\mathrm{al}^{29}$ ). 
TABLE III

BPHV Aortic Wall Lipid Content after $80 \%$ Ethanol Pretreatment, Pretreatment Time $24 \mathrm{~h}$

\begin{tabular}{lcc}
\hline $\begin{array}{c}\text { Pretreatment } \\
\text { Condition }\end{array}$ & $\begin{array}{c}\text { Cholesterol } \\
\text { (nmol/mg } \\
\text { Dry Weight) }\end{array}$ & $\begin{array}{c}\text { Phospholipids } \\
\text { (nmol/mg } \\
\text { Dry Weight) }\end{array}$ \\
\hline HEPES buffer, control & $8.97 \pm 0.33$ & $10.63 \pm 1.03$ \\
$80 \%$ Ethanol & $0.13 \pm 0.03$ & $0.6 \pm 0.0$ \\
\hline
\end{tabular}

All values are reported as average $\pm \operatorname{SEM}(n=3)$.

calcification. ${ }^{12}$ Bisphosphonate and ferric chloride $\left(\mathrm{FeCl}_{3}\right)$ sustained release was also inhibitory. ${ }^{13}$ Chen and coworkers, in fact, demonstrated that synergistic inhibition of aortic wall calcification was possible by combining amino oleic acid pretreatment with ferric chloride pretreatment. $^{14}$ Thus, ethanol pretreatment could be combined with a synergistic approach such as aluminum chloride pretreatment to inhibit both bioprosthetic aortic cusp calcification and bioprosthetic aortic wall calcification.

We have shown that ethanol pretreatment changes the collagen conformation in BPHV cusps as demonstrated by changes in the amide I region of the FTIR spectra. ${ }^{3}$ The interpretation of the spectra was straightforward due to the abundance of type I collagen in cusps. ${ }^{15}$ In the present work, however, the interpretation of changes in the amide I region is difficult because of the more complex nature of the aortic wall tissue. BPHV aortic wall tissue contains abundant elastin as well as collagen and other proteins exhibiting IR absorption at approximately the same frequencies. Structural changes in collagen have been extensively studied by FTIR, circular dichroism, and Raman spectroscopy, as reported in the literature. ${ }^{16-20}$ All the peaks observed in the FTIR for collagen are well assigned, ${ }^{16,17,19}$ and type I collagen has been confirmed to have a triple helical structure in the native state. ${ }^{16}$

However, elastin's tertiary structure is still a matter of investigation. Elastin has been postulated to have a mixture of alpha-helical and beta-sheet structures. ${ }^{21-24}$ Various studies have been reported for elastin structure determination by FTIR. ${ }^{21-24}$ A recent paper discusses the amide I region of FT-Raman spectra for elastin indicating the complex nature of this region. ${ }^{23}$ After deconvolution of the amide I band, the elastin's tertiary structure was determined to be a mixture of $10 \%$ alpha-helices, $45 \%$ beta-sheets, and $45 \%$ unidentified (turn + coil) conformations.

In our studies with aortic wall, a band at $1619 \mathrm{~cm}^{-1}$ in the amide I region could be ascribed to a beta-sheet structure for the elastin component. ${ }^{23}$ Disappearance of this band after ethanol pretreatment suggests a reduction in the proportion of beta-sheet regions of elastin as a consequence of ethanol pretreatment. A more complete interpretation of these changes is beyond the scope of the present studies, and will be the subject of future work. Ethanolic solutions have also been shown to increase the alpha-helical conformation of elastin as assessed by circular dichroism studies. ${ }^{22}$ Similar structural changes in elastin might be possible owing to our ethanol pretreatment protocol.

Proton NMR spin-lattice relaxation times $\left(T_{1}\right)$ have been used in the past for water structure and moisture content analysis, ${ }^{25}$ and have been applied to biomaterials to assess tissue-water interactions. ${ }^{6,7,26-28}$ In previous studies, ${ }^{29}$ we observed a marked decrease in the proton NMR relaxation time $\left(T_{1}\right)$ for glutaraldehydefixed cusps correlating with the concentration of the ethanol pretreatment solution (Fig. 5). This change in $T_{1}$ strongly suggested altered water interactions after the ethanol pretreatments. In comparison, the aortic wall $T_{1}$ values in the present study did not change with the ethanol pretreatment. This significant difference between the $T_{1}$ values for cusps and aortic wall after ethanol pretreatment reflects a markedly different water environment and may partly explain the differences in the anticalcification effects of ethanol for these two different tissues.

Lipid extraction data obtained in this study clearly demonstrate that ethanol pretreatment was highly efficacious in extracting phospholipids and cholesterol from the aortic wall tissue (Table III). Intrinsic phospholipids have been considered to be donors of phosphorus in the initial stages of mineralization. ${ }^{30} \mathrm{Al}-$ though the lipid extraction efficiency of ethanol pretreatment was greater for aortic wall tissue than cusps, ${ }^{3}$ the anticalcification effects were less than complete for aortic wall (as shown in this study), while ethanol pretreatment completely prevented cusp calcification. ${ }^{3}$ This observation also supports our view that lipid extraction is only part of the ethanol mechanism.

While calcification of aortic wall has not been demonstrated to cause prosthesis failure in the clinical setting, this consideration is nevertheless of great concern because of the emergence and widespread use of stentless bioprosthetic heart valves. ${ }^{31}$ Stentless bioprostheses have advantages compared to stentmounted heterograft valves in terms of the ability to implant bioprostheses in otherwise anatomically restrictive sites, as well as the ability to trim and tailor bioprosthetic implants according to the anatomy at the time of surgery. ${ }^{32}$ These considerations are particularly important in congenital heart surgery. Stentless valve replacements have also been viewed by many to be more advantageous for aortic valve replacement surgery, and even mitral valve replacement surgery. ${ }^{4}$ Although aortic wall calcification has not been observed to be a factor as yet in stentless valve clinical failure, aortic wall calcific deposits have been noted to be a major cause of failure in the use of aortic valve homografts. ${ }^{33}$ Excrescent calcific deposits on the aortic wall significantly interfered with the function of hu- 
man aortic homografts in clinical use for congenital heart surgery, ${ }^{34}$ and thus, it is reasonable to expect that the same pathophysiology in a stentless valve could be of similar importance in valve replacement surgery. Optimizing the ethanol inhibition of aortic wall calcification or finding a synergistic solution for complete inhibition could prove to be of great importance for using this general inhibitory approach in the clinical setting for stentless valves.

\section{CONCLUSIONS}

Ethanol pretreatment significantly inhibits porcine bioprosthetic aortic wall calcification in both subcutaneous BPHV implants and BPHV mitral valve replacements. However, ethanol inhibition of aortic wall calcification, while significant, is only partially effective. The mechanism of ethanol inhibition of BPHV aortic wall calcification is incompletely understood, but may be caused in part by lipid extraction and protein structural changes. However, ethanol pretreatment had no effect on proton NMR relaxation times for BPHV aortic wall in contrast to BPHV cusps; this may explain the less than complete inhibition of calcification.

\section{References}

1. F. J. Schoen, R. J. Levy, S. L. Hilbert, and R. W. Bianco, “Antimineralization treatments for bioprosthetic heart valves: Assessment of efficacy and safety," J. Thorac. Cardiovasc. Surg., 104, 1285-1288 (1992).

2. W. Chen, F. J. Schoen, and R. Levy, "Mechanism of efficacy of 2-amino oleic acid for inhibition of calcification of glutaraldehyde-pretreated porcine bioprosthetic heart valves," Circulation, 90, 323-329 (1994).

3. N. Vyavahare, D. Hirsh, E. Lerner, J. Baskin, F. J. Schoen, R. Bianco, H. S. Kruth, R. Zand, and R. J. Levy, "Prevention of bioprosthetic heart valve calcification by ethanol preincubation: Efficacy and mechanism," Circulation, 95, 479-488 (1997).

4. F. J. Schoen, D. Hirch, R. W. Bianco, and R. J. Levy, “Onset and progression of calcification in porcine aortic bioprosthetic valves implanted as orthotopic mitral valve replacements in juvenile sheep," J. Thorac. Cardiovasc. Surg., 108, 880-887 (1994).

5. P.S. Chen, "Microdetermination of phosphorous," Anal. Chem., 28, 1236-1238 (1956).

6. R. Kamman, K. Go, G. Stomp, C. Hulstaert, and H. Berendsen, "Changes in relaxation times T1 and T2 in rat tissues after biopsy and fixation," Magn. Reson. Imaging, 3, 245-250 (1985).

7. T. Scholz, S. Fleagle, and D. Skorton, "In vitro NMR characterization of mammalian myocardium: Effect of specimen integrity on relaxation times," Magn. Reson. Imaging, 11, 367-370 (1989).

8. J. Folch, M. Lees, and G. H. Sloane-Stanley, "A simple method for the isolation and purification of total lipids from animal tissues," J. Biol. Chem., 226, 497-509 (1957).

9. W. Gamble, M. Vaughan, H. S. Kruth, and J. Avigan, "Procedure for determination of free and total cholesterol in micro- or nanogram amounts suitable for studies with cultured cells," $J$. Lipid Res., 19, 1068-1070 (1978).
10. G. R. Bartlett, "Phosphorus assay in column chromatography," J. Biol. Chem., 234, 466-468 (1959).

11. M. Jackson, L.-P. Choo, P. H. Watson, W. C. Halliday, and H. H. Mantsch, "Beware of connective tissue proteins: Assignment and implications of collagen absorptions in infrared spectra of human tissues," Bichim. Biophys. Acta, 1270, 1-6 (1995).

12. C. Webb, N. M. Nguyen, F. J. Schoen, and R. J. Levy, "Calcification of allograft aortic wall in a rat subdermal model," Am. J. Pathol., 141, 487-496 (1992).

13. N. R. Vyavahare, X. Qu, M. Lee, P. Behari, F. J. Schoen, and R. J. Levy, "Synergism of calcium-ethanehydroxybisphosphate (CaEHBP) and $\mathrm{FeCl}_{3}$ : Controlled release polymers for preventing calcification of bioprosthetic aortic wall," J. Control. Rel., 34, 97-108 (1995).

14. W. Chen, F. J. Schoen, and R. Levy, "Synergistic inhibition of calcification of porcine aortic root with preincubation in $\mathrm{FeCl} 3$ and amino oleic acid in a rat subdermal model," J. Biomed. Mater. Res., 38, 43-48 (1997).

15. P. Mannschott, D. Herbage, M. Weiss, and C. Bufevant, "Collagen heterogenity in pig heart valves," Biochim. Biophys. Acta, 434, 177-183 (1976).

16. B. B. Doyle, E. G. Benedit, and E. R. Blout, "Infrared spectroscopy of collagen and collagen like peptides," Biopolymers, 14, 937-957 (1975).

17. A. George and A. Veis, "FTIRS in $\mathrm{H}_{2} \mathrm{O}$ demonstrates that collagen monomers undergo a conformational transition prior to thermal self-assembly in vitro," Biochemistry, 30, 2372-2377 (1991).

18. D. Jennes, C. Sprecher, and W. Johnson, Jr., "Circular dichroism of collagen, gelatin and poly(proline) II in the vacuum ultraviolet," Biopolymers, 15, 513-521 (1976).

19. V. Renugopalkrishnan, G. Chandrakasan, S. Moore, T. Hutson, C. Berney, and R. Bhatnager, "Bound water in collagen: Evidence from Fourier transform infrared and Fourier transform infrared photoacoustic spectroscopic study," Macromolecules, 22, 4121-4124 (1989).

20. H. Susi, J. Ard, and R. Carroll, "The infrared spectrum and water binding of collagen as a function of relative humidity," Biopolymers, 10, 1597-1604 (1971).

21. A. Tamburro, V. Guantieri, and D. Gordini, "Synthesis and structural studies of a pentapeptide sequence of elastin, poly(Val-Gly-Gly-Leu-Gly),"' J. Biomol. Struct. Dynam., 10, 441-454 (1992).

22. M. Mammi, L. Gotte, and G. Pezzin, "Evidence for order in the structure of alpha-elastin," Nature, 220, 371-373 (1968).

23. L. Debelle, A. Alix, M.-P. Jacob, J.-P. Huvenne, M. Berjot, B. Sombret, and P. Legrand, "Bovine elastin and K-elastin secondary structure determination by optical spectroscopies," $J$. Biol. Chem., 270, 26099-26103 (1995).

24. L. Debelle and A. Alix, "Optical spectroscopic determination of bovine tropoelastin molecular model," J. Mol. Struct., 348, 321324 (1995).

25. S. Yoshioka, Y. Aso, T. Otsuka, and S. Kojima, “Water mobility in poly(ethylene glycol)-, poly(vinylpyrrolidone)-, and gelatinwater," Pharmacol. Sci., 84, 1072-1077 (1995).

26. P. Bottomley, T. Foster, R. Argersinger, and L. Pfeifer, "A review of normal tissue hydrogen NMR relaxation times and relaxation mechanisms from 1-100 MHZ: Dependence on tissue type, NMR frequency, temperature, species, excision and age," Med. Phys., 11, 425 (1984).

27. S. F. Akber, "A new perspective to assess relaxation time of tumor," Med. Hypoth., 37, 24-26 (1992).

28. S. F. Akber, "Role of paramagnetic ions and water proton spinlattice relaxation time in biological systems," Nuklearmedizin, 32, 52-56 (1993).

29. N. Vyavahare, D. Hirsh, E. Lerner, J. Baskin, F. J. Schoen, R. 
Bianco, H. S. Kruth, R. Zand, and R. J. Levy, "Prevention of calcification of glutaraldehyde-crosslinked porcine aortic cusps by ethanol preincubation: Mechanistic studies concerning protein structure and water-biomaterial relationships," $J$. Biomed. Mater. Res., 40, 577-585 (1998).

30. F. J. Schoen, H. Harasaki, K. Kim, H. C. Anderson, and R. J. Levy, "Biomaterial associated calcification: Pathology, mechanisms, and strategies for prevention," J. Biomed. Mater. Res., 22, 11-36 (1988).

31. D. N. Kon, S. Westaby, N. Amarasena, R. Pillai, and A. R. Cordell, "Comparison of implantation techniques using free- style stentless porcine aortic valve," Ann. Thorac. Surg., 59, 857862 (1995).

32. W. Konertz, P. Hamann, E. Schwammenthal, G. Breithardt, and H. H. Scheld, "Aortic valve replacement with stentless xenografts," J. Heart Valve Dis., 1, 249-252 (1992).

33. D. C. Cleveland, W. G. Williams, and A. J. Razzouk, "Failure of cryopreserved homograft valved conduits in the pulmonary circulation," Circulation, 86(Suppl. 5), 150-153 (1992).

34. B. G. Barratt-Boyes, "Homograft aortic valve replacement in aortic valve replacement in aortic incompetence and stenosis," Thorax, 19, 131-150 (1964). 